\title{
Usabilidade e acessibilidade no desenvolvimento de interfaces para ambientes de educação à distância
}

\author{
Patrícia B. Scherer Bassani, Universidade Feevale, patriciab@feevale.br \\ Patricia Alejandra Behar, UFRGS, pbehar@terra.com.br \\ Regina de Oliveira Heidrich, Universidade Feevale, rheidrich@feevale.br \\ Alan Bittencourt, PIBIC CNPq/Feevale, abittencourt@feevale.br \\ Eliane Ortiz, Universidade Feevale, elianyortiz@gmail.com
}

\begin{abstract}
Resumo: Na educação a distância (EAD) a mediação didático-pedagógica do processo de ensino-aprendizagem é realizada a partir das tecnologias da informação e comunicação. Isto permite que professores e alunos desenvolvam atividades em lugares e/ou tempo diversos. Esta flexibilidade reduz a necessidade de locomoção e facilita o envolvimento de pessoas com deficiência em atividades educativas. Torna-se relevante, então, a discussão sobre as possibilidades de utilização dos ambientes de EAD por pessoas com diferentes necessidades, físicas e/ou cognitivas. Assim, este artigo busca refletir sobre a aplicabilidade do Design Universal e do Design de Interação no desenvolvimento de interfaces para EAD. O estudo aponta como o projeto de interfaces deveria articular os conceitos de usabilidade, acessibilidade e experiência do usuário.
\end{abstract}

Palavras-chave: educação a distância; usabilidade; acessibilidade; interface.

Title: Usability and accessibility in the development of interfaces for distance learning environments.

\begin{abstract}
In distance education the didactic and pedagogical mediation of the learning process occurs through information and communication technologies. This allows teachers and students to develop activities in different spaces and times. This flexibility reduces the necessity of physical locomotion and makes it possible for disabled people to get involved in educational activities. This way, the discussion about the possibilities of the use of distance learning environments by people with disabilities, whether physical or cognitive, becomes relevant. Our paper, then, presents a reflection on the application of the Universal Design and Interaction Design in the development of interfaces for distance learning. The study points out that the interface project must articulate the concepts of usability, accessibility and user experience.
\end{abstract}

Keywords: distance learning; usability; accessibility; interface.

\section{Introdução}

De acordo com a legislação brasileira, a educação a distância (EAD) é uma "modalidade educacional na qual a mediação didático-pedagógica nos processos de ensino e aprendizagem ocorre com a utilização de meios e tecnologias de informação e comunicação, com estudantes e professores desenvolvendo atividades educativas em lugares ou tempos diversos” (DECRETO N 5.622).

Conforme o documento intitulado "Referenciais de qualidade para a educação superior à distância” (MEC, 2009), material elaborado pelo Ministério da Educação/Secretaria de Educação a Distância, a partir de uma discussão pública com 
especialistas na área e universidades, "o uso inovador da tecnologia aplicada à educação deve estar apoiado em uma filosofia de aprendizagem que proporcione aos estudantes efetiva interação no processo de ensino-aprendizagem”. E ainda, "o princípio da interação e da interatividade é fundamental para o processo de comunicação e devem ser garantidos no uso de qualquer meio". De acordo com o referido documento, deve ser privilegiada e garantida a interação entre professor-aluno, professor-tutor e tutor-aluno, e a relação entre colegas de curso deve ser fomentada.

A legislação também prevê que os projetos pedagógicos de cursos e programas na modalidade à distância deverão disponibilizar atendimento apropriado aos estudantes com necessidades especiais. Assim, em uma proposta de educação à distância, sujeitos com diferentes necessidades, sejam elas físicas e/ou cognitivas, temporárias ou permanentes, compartilham o mesmo ambiente virtual de aprendizagem.

Um ambiente virtual de aprendizagem (AVA) é caracterizado por um conjunto de ferramentas computacionais que permitem a criação e o gerenciamento de cursos à distância, potencializando processos de interação, colaboração e cooperação (BASSANI, 2006).

Estudos apontam que a interface de um AVA pode potencializar a interatividade e a interação no processo educacional a distância (MACHADO JUNIOR, 2008).

Johnson (2001) entende que a interface se refere a softwares que “dão forma” à interação entre usuário e sistema. Para ele, "a interface atua como uma espécie de tradutor, mediando as duas partes, tornando uma sensível á outra” (p. 17). Rocha e Baranauskas (2003, p. 13), afirmam que "não se pode pensar em interfaces sem considerar o ser humano que vai usá-la e, portanto, interface e interação são conceitos que não podem ser estabelecidos ou analisados independentemente”.

Nessa perspectiva, considerando-se que os ambientes de EAD devem possibilitar a interação entre diferentes sujeitos, um primeiro desafio consiste em permitir o acesso e a participação, independente da condição deste sujeito, deficiente ou não. Para tanto, entende-se que a discussão sobre aprendizagem na modalidade à distância deve também nortear estudos na área de desenvolvimento de interfaces acessíveis.

Neste estudo, entende-se que uma interface acessível deve ser pautada nos conceitos de acessibilidade e usabilidade. Entende-se a acessibilidade como a possibilidade de acesso ao AVA, independente das diferentes necessidades e/ou deficiências do sujeito. Por outro lado, a usabilidade consiste em que este ambiente seja fácil de usar, eficiente e agradável, da perspectiva do sujeito-usuário.

Assim, este artigo busca refletir sobre os conceitos que norteiam o desenvolvimento de interfaces para educação à distância. O presente estudo, de abordagem qualitativa, parte de uma reflexão sobre Design da Interação e Design Universal, para posteriormente delinear uma proposta para o desenvolvimento de interfaces para ambientes virtuais de aprendizagem.

\section{Design de Interação}

O Design de Interação (DI) é uma área de pesquisa caracterizada pela interconexão de diferentes campos interdisciplinares, como interação humano-computador (IHC), ergonomia cognitiva, trabalho cooperativo apoiado por computador (computer supported cooperative work - CSCW) entre outras (PREECE et al., 2005).

Conforme Preece et al. (2005, p. 28) o design da interação implica em "criar experiências que melhorem e estendam a maneira como as pessoas trabalham, se 
comunicam e interagem”. Também pode ser entendido como "o projeto de espaços para comunicação e interação humana".

As autoras apontam que o DI prevê a implementação de metas de usabilidade e metas decorrentes da experiência do usuário.

A usabilidade é considerada "o fator que assegura que os produtos sejam fáceis de usar, eficientes e agradáveis, da perspectiva do usuário” (PREECE et al., 2005, p. 35). Usabilidade implica na otimização das interações estabelecidas pelas pessoas com produtos interativos, a fim de possibilitar que elas realizem suas atividades no trabalho, na escola ou em atividades de lazer, sendo dividida nas seguintes metas:

a) eficiência: cumprir os objetivos para o qual foi criada;

b) segurança: permitir o manuseio de forma segura para o usuário e para o próprio sistema;

c) utilidade: ser útil para o usuário e as atividades que ele pretende desempenhar; usuário;

d) capacidade de aprendizagem: ser uma interface de fácil aprendizado para o

e) capacidade de memorização: ser fácil de lembrar como se usa.

Além das metas de usabilidade, a expansão do uso das tecnologias da informação e comunicação pelo público em geral impulsionou diferentes metas decorrentes da experiência do usuário. Assim, o DI está cada vez mais preocupado com a criação de sistemas que sejam satisfatórios, agradáveis, divertidos, interessantes, úteis, motivadores, esteticamente apreciáveis, incentivadores de criatividade, entre outros. De acordo com Preece et al. (2005), é importante que exista um equilíbrio entre as metas de usabilidade e as decorrentes da experiência do usuário.

Tullis et al. (2008) afirmam que a experiência do usuário faz referência a todos aspectos da interação entre um determinado sujeito e um produto, uma aplicação ou sistema. Os autores entendem que as métricas de usabilidade podem revelar algo sobre a experiência do usuário: aspectos relacionados à eficácia (ser capaz de completar uma tarefa), a eficiência (a quantidade de esforço necessário para concluir a tarefa), ou satisfação (o grau em que o usuário estava feliz com a sua experiência ao executar a tarefa).

De acordo com Tullis et al. (2008) é possível medir a experiência do usuário a partir de métricas de usabilidade, como por exemplo, número de cliques, tempo de resposta, tempo de realização de tarefas, quantidade de erros dos usuários ao tentar fazer login num sistema, etc. Entretanto, os autores destacam que existe diferença entre os termos usabilidade e experiência do usuário ou user experience (UX).

Usabilidade, conforme a ISO 9241-11, é a extensão na qual um produto pode ser "utilizado por usuários específicos para alcançar objetivos específicos com eficácia, eficiência e satisfação, num contexto de uso específico". Por outro lado, a UX é usualmente considerada a habilidade do usuário em usar o produto ou sistema para realizar a tarefa com sucesso; envolve a interação entre o sujeito e o objeto (produto ou sistema), assim como pensamento, sentimentos e percepções que resultam das interações.

Todas as métricas de usabilidade devem ser observáveis de alguma forma (direta ou indireta), devem ser quantificáveis e devem representar a UX num formato numérico (por exemplo: 65\% dos usuários estão satisfeitos com o produto, 90\% dos usuários são capazes de completar um conjunto de tarefas em menos de um minuto). 
Rubin e Chisnell (2008) apontam que, para ser usável, um produto ou serviço deve ser útil, eficiente, eficaz, satisfatório, fácil de aprender e acessível.

A utilidade diz respeito ao grau em que um produto permite ao usuário atingir seus objetivos e é uma avaliação da facilidade do usuário em utilizar o produto. A eficiência é a rapidez com que o objetivo do usuário pode ser realizado de forma precisa, e é geralmente uma medida de tempo (por exemplo: 95\% de todos os usuários serão capazes de...). A eficácia consiste em verificar se o produto se comporta da mesma forma que os usuários esperam e a facilidade com que os usuários podem usá-lo para fazer o que eles pretendem. O potencial de aprendizagem é uma parte da eficácia e tem a ver com a capacidade do usuário para operar o sistema com algum nível de competência, após período de capacitação/formação. Refere-se, também, à capacidade dos usuários pouco frequentes em reaprender o sistema após períodos de inatividade. A satisfação refere-se à percepção, sentimentos e opiniões do usuário em relação ao produto, geralmente capturados por entrevistas.

Rubin e Chisnell (2008) destacam a questão da acessibilidade como um critério de avaliação da usabilidade, que implica em possibilitar acesso a pessoas com deficiência. Para os autores, fazer um produto usável por pessoas com deficiência quase sempre beneficia também as pessoas sem deficiência (isto inclui deficiências permanentes ou temporárias). Esta concepção reflete as idéias do Design Universal, apresentado na seção a seguir.

\section{Design Universal}

A terminologia Universal Design (Design Universal) foi criada, em 1987, pelo americano Ron Mace, arquiteto que usava cadeira de rodas e um respirador artificial. Segundo Carletto e Cambiaghi (2008), "Mace acreditava que esse era o surgimento não de uma nova ciência ou estilo, mas a percepção da necessidade de aproximarmos as coisas que projetamos e produzimos, tornando-as utilizáveis por todas as pessoas".

O conceito de Design Universal (DU) se desenvolveu entre profissionais de Design e Arquitetura, principalmente na Universidade da Carolina do Norte (EUA), objetivando o projeto de ambientes e produtos para serem usados por todos sem a necessidade de adaptação ou projeto especializado para pessoas com deficiência (Center for Universal Design).

Então, na década de 90, Ron Mace criou um grupo com arquitetos e estudiosos da área de acessibilidade para estabelecerem os sete princípios do DU, que são usados mundialmente em projetos de acessibilidade (CARLETTO, CAMBIAGHI, 2008). São eles:

a) uso equiparável: o produto/ambiente deve poder ser utilizado por pessoas com diferentes capacidades;

b) uso flexível: deve atender pessoas com diferentes habilidades e preferências, sendo adaptável para qualquer uso;

c) uso simples e intuitivo: de fácil entendimento, independente do conhecimento do usuário, habilidades de linguagem ou limitações perceptivas;

d) informação de fácil percepção: a informação tem que ser transmitida de forma a atender as necessidades do receptor, seja ele estrangeiro ou com limitações perceptivas;

e) tolerante a erros: devem-se minimizar os riscos e possíveis conseqüências de ações não intencionais ou acidentais; 
f) baixo esforço físico: o ambiente tem de ser usado eficientemente, com conforto e com o mínimo de desgaste físico;

g) dimensão e espaço para aproximação e uso: devem-se estabelecer dimensões e espaços apropriados para o acesso, o alcance, a manipulação e o uso, independentemente do tamanho do corpo, da postura ou mobilidade do usuário.

Estes sete princípios do Design Universal foram criados para ambientes e produtos, tanto físicos quanto virtuais, com a intenção de torná-los utilizáveis por todas as pessoas. Conforme a NBR 9050:2004, elaborada pela Associação Brasileira de Normas Técnicas (ABNT), desenho universal é "aquele que visa atender á maior gama de variações possíveis das características antropométricas e sensoriais da população".

Assim, percebe-se que o conceito de acessibilidade está intimamente relacionado ao conceito de DU. A Lei $\mathrm{n}^{0}$ 10.098/00, que estabelece normas gerais e critérios básicos para a promoção da acessibilidade das pessoas com deficiência ou com mobilidade reduzida, define acessibilidade como "a possibilidade e condição de alcance para utilização, com segurança e autonomia, dos espaços, mobiliários e equipamentos urbanos, das edificações, dos transportes e dos sistemas e meios de comunicação por pessoa portadora de deficiência ou com mobilidade reduzida". Conforme a referida lei, existem quatro diferentes barreiras (ou obstáculos) que limitam ou impedem o acesso, a liberdade de movimento e a circulação com segurança das pessoas: barreiras arquitetônicas urbanísticas; barreiras arquitetônicas na edificação; barreiras arquitetônicas nos transportes; barreiras nas comunicações.

Com a expansão da Internet, surgiu também a necessidade de prover acesso universal na Web com a construção de ambientes virtuais acessíveis. Isto impulsionou a criação de consórcios mundiais, como W3C (World Wide Web Consortium) e a WAI (Web Accessibility Initiative), explicitados na próxima seção.

\section{Acessibilidade e usabilidade na web}

Conforme Berners-Lee, diretor da W3C ${ }^{1}$ e inventor da World Wide Web, “o poder da Web está em sua universalidade. Acesso por todos, independentemente da deficiência é um aspecto essencial ${ }^{2}$.

O W3C prevê padrões (standards) para acessibilidade na web, uma vez que esta foi projetada para funcionar para todas as pessoas, independentemente do seu hardware, software, língua, cultura, localização, capacidade física ou mental.

Conforme o censo demográfico realizado em 2000, pelo Instituto Brasileiro de Geografia e Estatística (IBGE), aproximadamente 24,6 milhões de pessoas, ou 14,5\% da população total, apresentaram algum tipo de incapacidade ou deficiência (física, auditiva, visual, mental ou deficiências múltiplas). O desenvolvimento de sites a partir dos padrões W3C diminui o impacto da deficiência, porque a Web remove as barreiras de comunicação e de interação que muitas pessoas enfrentam no mundo físico.

O documento Web Content Accessibility Guidelines (WCAG) 2.0, ou Diretrizes de Acessibilidade para Conteúdo Web, define a forma de tornar o conteúdo Web mais acessível às pessoas com deficiência. Quatro princípios fornecem a base para a acessibilidade da Web:

\footnotetext{
${ }^{1} \mathrm{O}$ World Wide Web Consortium (W3C) é uma comunidade internacional que desenvolve padrões para garantir para garantir o crescimento a longo prazo da Web.

2 "The power of the Web is in its universality. Access by everyone regardless of disability is an essential aspect" (BERNERS-LEE, Tim).
} 
a) perceptível: os usuários devem ser capazes de perceber a informação que está sendo apresentada (ela não pode ser invisível a todos os seus sentidos);

b) operável: os usuários devem ser capazes de operar a interface; a interface não pode exigir a interação que um usuário não pode executar;

c) compreensível: os usuários devem ser capazes de compreender as informações, bem como o funcionamento da interface do usuário;

d) robusto: os usuários devem ser capazes de acessar o conteúdo de forma confiável por uma ampla variedade de agentes de usuário, incluindo tecnologias assistivas.

Esses princípios orientam diretrizes para que autores/desenvolvedores possam tornar os conteúdos mais acessíveis para usuários com diferentes deficiências. Assim, o W3C constitui importante referência e orientação para o desenvolvimento de sites acessíveis.

Estudos na área de design da navegação (KALBACH, 2009) apontam a acessibilidade como um princípio que deve estar sempre presente no design para a web. Para o autor, "a acessibilidade web comumente refere-se a construir sites de forma que pessoas com necessidades especiais possam usá-lo" (p. 10). Destaca, ainda, que "sites que são acessíveis possuem benefícios para outros, também” (p. 10). Assim, as questões relativas à acessibilidade não podem ser previstas apenas no fim do projeto e também não se encaixam em uma fase ou atividade, devem permear todo o processo de design de navegação.

Quando se trata da usabilidade na Web, percebe-se a inexistência de uma padronização. Entretanto, a usabilidade é uma das características relacionadas à qualidade de software. A norma ISO/IEC 25030:2008 ${ }^{3}$ define a usabilidade como " $a$ capacidade do produto de software em ser compreendido, aprendido, usado e atraente ao usuário, quando utilizado em condições especificadas”. Nessa perspectiva, a usabilidade de um produto de software deve ser avaliada a partir das especificações do sistema.

Existem diferentes propostas para avaliar a usabilidade. Conforme Dias (2007), dentre os diferentes métodos destaca-se a avaliação heurística. Neste método os princípios utilizados para a avaliação são as heurísticas de Nielsen ${ }^{4}$, listadas abaixo:

a) visibilidade do estado atual do sistema: manter o usuário informado do que está acontecendo no sistema;

b) correlação entre o sistema e o mundo real: o sistema deve falar a linguagem do usuário;

c) controle e liberdade do usuário: deve ser possível ao usuário desfazer ou refazer operações do sistema;

d) consistência e padrões: palavras ou ações diferentes que tenham a mesma função no sistema não devem causar confusão no usuário;

e) prevenção de erros: o sistema tem que ser projetado cuidadosamente para prevenir a ocorrência de erros;

\footnotetext{
${ }^{3}$ Esta norma fornece os requisitos e recomendações para especificação de requisitos de qualidade de produto de software

${ }^{4}$ Estas foram desenvolvidas em 1990 com a colaboração de Molich. Em 1994 Nilsen analisou fatores de 249 problemas de usabilidade, e a partir deste estudo condensou esses problemas em dez heurísticas de usabilidade (DIAS, 2007).
} 
f) reconhecimento ao invés de memorização: as instruções de uso do sistema devem estar visíveis ou facilmente acessíveis quando necessário;

g) flexibilidade e eficiência de uso: usuário pode personalizar ou programar ações freqüentes;

h) projeto estético e minimalista: não deve conter informações irrelevantes ou raramente necessárias;

i) suporte aos usuários no recolhimento, diagnóstico e recuperação de erros: mensagens de erro devem ser claras, mostrando precisamente o problema e sugerindo soluções;

j) informações de ajuda e documentação: a documentação do sistema deve sempre estar disponível ao usuário.

Baseando-se nos estudos teóricos apresentados até aqui, na seção seguinte busca-se delinear uma proposta para o desenvolvimento de interfaces para educação à distância, que possa contemplar os diferentes públicos que vêm utilizando a Internet como forma de socialização e acesso à educação.

\section{0 desenvolvimento de interfaces sob a perspectiva da acessibilidade, usabilidade e experiência do usuário}

Para Badre (2002), os mesmos princípios básicos que regem o design de interfaces de software se aplicam para a concepção de web sites e aplicações web. Assim como uma interface mal projetada pode prejudicar um software apesar da sua funcionalidade complexa ou o poder da sua tecnologia, uma interface Web inadequada, apesar dos seus gráficos impressionantes, pode impulsionar o usuário para outro site com um clique do mouse.

Nos seus estudos sobre a interface de AVA, Machado Junior (2008) entende que "do ponto de vista da interface gráfica, a abordagem centrada no usuário é que diferencia os AVAs interativos baseados na web dos web sites comerciais, institucionais e portais da web" (p. 90). O autor destaca que embora um AVA se diferencie de um web site, especialmente no que se refere aos objetivos, programação e ferramentas disponíveis, ambos compartilham a mesma tecnologia de suporte na Web. Assim, "quando são feitas considerações sobre a interface gráfica de um AVA, cuja operação e acesso efetuam-se pela World Wide Web, é preciso que se considerem aspectos de design visual aplicados em web sites" (p. 90).

Assim, a partir dos estudos realizados, entende-se que uma proposta para o desenvolvimento de interfaces acessíveis para EAD deve articular estudos na área de Design Universal e Design da Interação, conforme figura 1.

A proposta de Design Universal contempla o conceito de acessibilidade a partir de princípios norteadores que orientam o desenvolvimento de produtos (objetos em geral ou software). Por outro lado, o Design de Interação enfatiza a usabilidade, por meio de cinco metas. Para as questões relacionadas à experiência do usuário, não existem parâmetros específicos, eles devem ser obtidos por meio de entrevistas, questionários ou monitoramento das ações do sujeito no ambiente. As heurísticas de Nielsen (2000) constituem referencial relevante para a reflexão sobre usabilidade. 


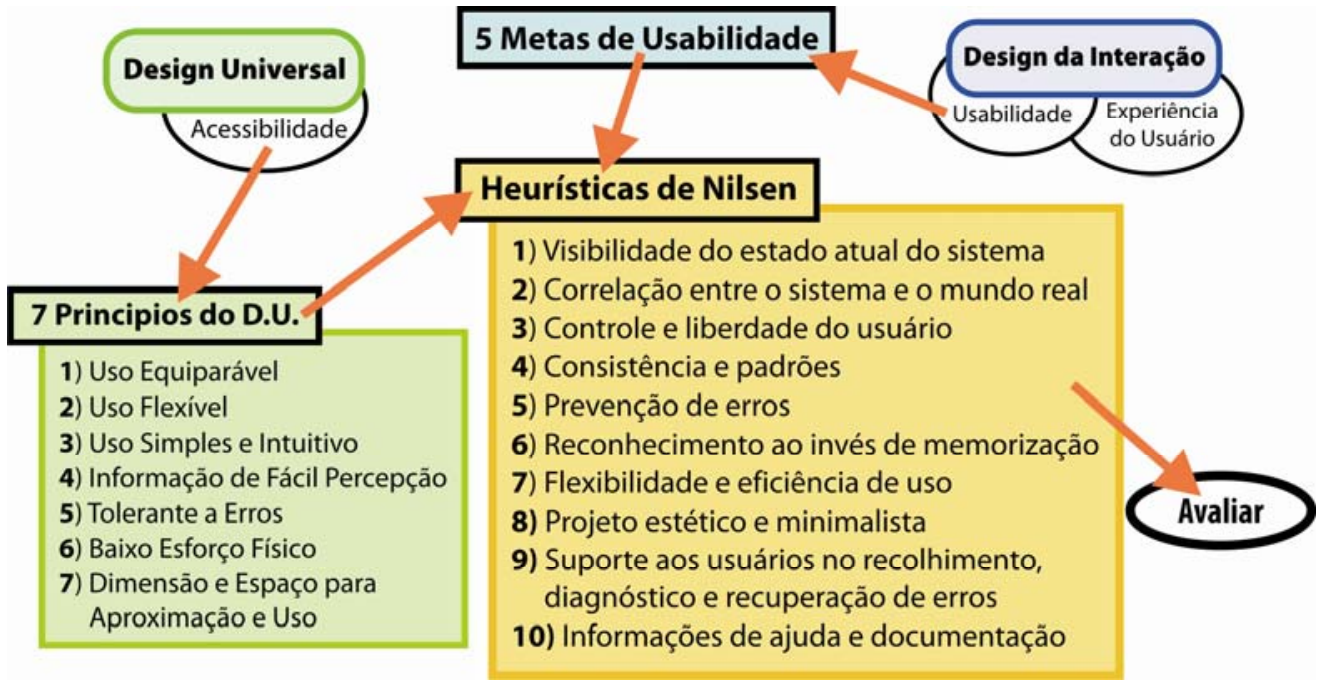

Figura 1 - Diretrizes para o desenvolvimento de interfaces acessíveis Fonte: autores do artigo

Assim, os princípios do DU são entendidos como diretrizes gerais de acessibilidade que devem orientar a reflexão sobre usabilidade. Nessa perspectiva, cada princípio de usabilidade (heurísticas) deve ser pensado/projetado à luz dos princípios de acessibilidade (ou DU). Após a definição conceitual, partiu-se para a especificação dos parâmetros que devem guiar o desenvolvimento de interfaces para ambientes de EAD. Estes estão descritos na tabela 1.

Tabela 1 - Parâmetros para o desenvolvimento de interfaces

\begin{tabular}{|c|c|c|c|c|c|c|c|}
\hline $\begin{array}{c}\text { Heurística/Desig } \\
\text { n Universal }\end{array}$ & $\begin{array}{c}\text { Uso } \\
\text { equiparável }\end{array}$ & Uso flexível & $\begin{array}{c}\text { Uso } \\
\text { simples e } \\
\text { intuitivo }\end{array}$ & $\begin{array}{l}\text { Informação de } \\
\text { fácil percepção }\end{array}$ & $\begin{array}{l}\text { Tolerante a } \\
\text { erros }\end{array}$ & $\begin{array}{l}\text { Baixo } \\
\text { esforço } \\
\text { físico }\end{array}$ & $\begin{array}{l}\text { Dimensão e } \\
\text { espaço para } \\
\text { aproximação } \\
\text { e uso }\end{array}$ \\
\hline $\begin{array}{l}\text { Visibilidade do } \\
\text { estado atual do } \\
\text { sistema }\end{array}$ & $\begin{array}{c}\text { Permitir uso } \\
\text { com leitor de } \\
\text { tela e/ou } \\
\text { hardware } \\
\text { especial; } \\
\text { tempo de } \\
\text { carga da } \\
\text { interface; } \\
\text { vídeos com } \\
\text { legenda. }\end{array}$ & $\begin{array}{c}\text { Adaptação da } \\
\text { visualização na } \\
\text { tela } \\
\text { (aumentar/diminui } \\
\text { r fonte, cor de } \\
\text { letra/fundo, variar } \\
\text { resolução, } \\
\text { desabilitar som e } \\
\text { imagens, acessar } \\
\text { links e formulários } \\
\text { por meio da tecla } \\
\text { TAB) }\end{array}$ & $\begin{array}{l}\text { Padronizar } \\
\text { layout da } \\
\text { interface }\end{array}$ & & $\begin{array}{c}\text { Compatível } \\
\text { com } \\
\text { diferentes } \\
\text { navegadores } \\
\text { Web. }\end{array}$ & $\begin{array}{l}\text { Evitar } \\
\text { múltiplas } \\
\text { janelas } \\
\text { (recomendad } \\
\text { o é utilizar } \\
\text { abas) }\end{array}$ & $\begin{array}{c}\text { Ícones } \\
\text { devem ser } \\
\text { maiores que } \\
\text { o tamanho da } \\
\text { seta do } \\
\text { mouse, a fim } \\
\text { de facilitar } \\
\text { uso de } \\
\text { mouses } \\
\text { diferenciados } \\
\text {. }\end{array}$ \\
\hline $\begin{array}{l}\text { Controle e } \\
\text { liberdade do } \\
\text { usuário }\end{array}$ & & $\begin{array}{c}\text { Permitir } \\
\text { pesonalização de } \\
\text { comandos de } \\
\text { tecla, menus e } \\
\text { caixas de } \\
\text { diálogos; permitir } \\
\text { habilitar/desabilita } \\
\text { r animações } \\
\text { gráficas. }\end{array}$ & & & $\begin{array}{c}\text { Evitar perda } \\
\text { de dados do } \\
\text { usuário na } \\
\text { recarga da } \\
\text { página Web; } \\
\text { permitir a } \\
\text { correção de } \\
\text { campos } \\
\text { independente } \\
\text { s em } \\
\text { formulários; } \\
\text { permiitr } \\
\text { refazer ações. }\end{array}$ & $\begin{array}{l}\text { Fornecer } \\
\text { acesso com } \\
\text { um único } \\
\text { clique ao } \\
\text { maior número } \\
\text { de recursos } \\
\text { possíveis. }\end{array}$ & \\
\hline
\end{tabular}




\begin{tabular}{|c|c|c|c|c|c|c|c|}
\hline $\begin{array}{l}\text { Heurística/Desig } \\
\text { n Universal }\end{array}$ & $\begin{array}{c}\text { Uso } \\
\text { equiparável }\end{array}$ & Uso flexível & $\begin{array}{c}\text { Uso } \\
\text { simples e } \\
\text { intuitivo }\end{array}$ & $\begin{array}{l}\text { Informação de } \\
\text { fácil percepção }\end{array}$ & $\begin{array}{l}\text { Tolerante a } \\
\text { erros }\end{array}$ & $\begin{array}{l}\text { Baixo } \\
\text { esforço } \\
\text { físico }\end{array}$ & \begin{tabular}{|c|}
$\begin{array}{c}\text { Dimensão e } \\
\text { espaço para } \\
\text { aproximação } \\
\text { e uso }\end{array}$ \\
\end{tabular} \\
\hline $\begin{array}{c}\text { Consistência e } \\
\text { padrões }\end{array}$ & \multicolumn{7}{|c|}{\begin{tabular}{|l} 
Padronizar nomes de comandos/ações e links; padronizar ícones; o texto alternativo de imagens e os links devem ser \\
equivalentes, independente do hardware/software utilizado.
\end{tabular}} \\
\hline $\begin{array}{l}\text { Prevenção de } \\
\quad \text { erros }\end{array}$ & $\begin{array}{c}\text { Utilizar } \\
\text { padronizaçã } \\
\text { o W3C }\end{array}$ & $\begin{array}{c}\text { Compatível com } \\
\text { diferentes } \\
\text { navegadores } \\
\text { Web. }\end{array}$ & & & & & \begin{tabular}{|c|} 
Prederminar \\
a estrutura do \\
site, \\
posicionando \\
a localização \\
das imagens, \\
antes da \\
carga da \\
Web (para \\
viabilizar uso \\
correto de \\
leitor de tela) \\
\end{tabular} \\
\hline $\begin{array}{l}\text { Reconhecimento } \\
\text { ao invés de } \\
\text { memorização }\end{array}$ & \begin{tabular}{|c|} 
Cada ação \\
deve retornar \\
um feedback \\
(salvar, \\
alterar, \\
modificar). \\
\end{tabular} & & & $\begin{array}{l}\text { Informar a } \\
\text { localização do } \\
\text { sujeito no } \\
\text { ambiente } \\
\text { ("migalhas de } \\
\text { pão") }\end{array}$ & & & \\
\hline $\begin{array}{l}\text { Flexibilidade e } \\
\text { eficiência de uso }\end{array}$ & \multicolumn{7}{|c|}{ O princípio de Design Universal "Uso flexível" contempla esta heurística. } \\
\hline $\begin{array}{l}\text { Projeto estético e } \\
\text { minimalista }\end{array}$ & \begin{tabular}{|c|} 
Facilitar uso \\
pelo teclado, \\
organizando \\
a sequencia \\
de acesso \\
via tecla \\
TAB; \\
minimizar o \\
número de \\
links em \\
cada página, \\
para facilitar \\
o uso da \\
tecla TAB. \\
\end{tabular} & $\begin{array}{l}\text { Limitar a utilização } \\
\text { de rolagem de } \\
\text { tela. }\end{array}$ & $\begin{array}{l}\text { Design de } \\
\text { navegação }\end{array}$ & $\begin{array}{l}\text { Ordenar de forma } \\
\text { lógica a } \\
\text { navegação interna } \\
\text { da página. }\end{array}$ & & & \\
\hline $\begin{array}{l}\text { Suporte aos } \\
\text { usuários no } \\
\text { recolhimento, } \\
\text { diagnóstico e } \\
\text { recuperação de } \\
\text { erros }\end{array}$ & & & & $\begin{array}{l}\text { Centralizar } \\
\text { mensagens de } \\
\text { erro na tela; } \\
\text { proporcionar } \\
\text { acesso a } \\
\text { conteúdos/página } \\
\text { s de ajuda. }\end{array}$ & $\begin{array}{c}\text { Evitar } \\
\text { botões/links } \\
\text { previamente } \\
\text { selecionados, } \\
\text { para } \\
\text { minimizar } \\
\text { ações } \\
\text { involuntárias. }\end{array}$ & & \\
\hline $\begin{array}{l}\text { Informações de } \\
\text { ajuda e } \\
\text { documentação }\end{array}$ & $\begin{array}{c}\text { Todas as } \\
\text { telas deve } \\
\text { ter acesso } \\
\text { ao conteúdo } \\
\text { da ajuda. }\end{array}$ & $\begin{array}{l}\text { Permitir diferentes } \\
\text { formas de } \\
\text { visualização da } \\
\text { ajuda (salvar, } \\
\text { visualizar no site } \\
\text { ou imprimir). }\end{array}$ & & & & & \\
\hline
\end{tabular}

Entende-se que a especificação de interfaces a partir da perspectiva apontada neste estudo torna possível o desenvolvimento de interfaces para diferentes sujeitos, com diferentes necessidades especiais e específicas.

\section{Considerações finais}

A partir do levantamento teórico realizado para diferenciar os conceitos de acessibilidade e usabilidade, percebe-se que eles estão fortemente relacionados, mas enfatizados de forma diferenciada nos estudos sobre Design de Interação e Design Universal.

O Design de Interação enfatiza as métricas de usabilidade e experiência do usuário (UX), deixando para o desenvolvedor/projetista a possibilidade de optar pela aplicação de critérios de acessibilidade. Dessa forma, fica evidente que o Design de 
Interação, por si só, não dá conta de atender a todos os públicos que hoje se fazem presente na Internet. Por outro lado, também o Design Universal não contempla todos os conceitos necessários para a real inclusão de todos os públicos, pois enfatiza essencialmente critérios de acessibilidade.

Nesse sentido, percebe-se a necessidade de estruturar um projeto de interfaces no qual os três conceitos (usabilidade, acessibilidade e UX) sejam articulados, a fim de permitir a construção de interfaces para ambientes de EAD, que realmente atendam as necessidades dos diferentes públicos que vêm utilizando a Internet, como forma de socialização e acesso a educação.

\section{Referências}

ASSOCIAÇÃO BRASILEIRA DE NORMAS TÉCNICAS. Norma NBR 9050: Acessibilidade a Edificações, Mobiliário, Espaços e Equipamentos Urbanos. Rio de Janeiro, 2004.

BADRE, Albert N. Shaping Web Usability: Interaction Design in Context. Boston, MA, USA: Addison-Wesley Professional, 2002

BASSANI, Patrícia B. S. Mapeamento das interações em ambiente virtual de aprendizagem: uma possibilidade para avaliação em educação a distância. Porto Alegre: PPGIE/UFRGS, 2006. Tese de Doutorado.

BERNERS-LEE, T. Longer Biography. Disponível em: <http://www.w3.org/People/ Berners-Lee/Longer.html>. Acesso em 23/06/2009.

CARLETTO, Ana Claudia; CAMBIAGHI, Silvana. Desenho Universal: um conceito para todos. São Paulo, 2008.

CENTER FOR UNIVERSAL DESIGN, College of Design, North Carolina State University. Disponível em < http://www.design.ncsu.edu/cud/index.htm>. Acesso em 15/02/2010.

DIAS, Cláudia. Usabilidade na web: criando portais mais acessíveis. $2^{\mathrm{a}}$ Ed., Rio de Janeiro: Alta Books, 2007.

JOHNSON, Steven. Cultura da interface: como o computador transforma nossa maneira de criar e comunicar. Rio de Janeiro: Jorge Zahar, 2001.

KALBACH. James. Design de navegação web: otimizando a experiência do usuário. Porto Alegre: Bookman, 2009.

MACHADO JUNIOR, Felipe S. Interatividade: a interface de um ambiente virtual de aprendizagem. Passo Fundo: IMED, 2008.

MEC. Referenciais de qualidade para a educação superior à distância. Disponível em: <http://portal.mec.gov.br/seed/arquivos/pdf/legislacao/refead1.pdf >. Acesso em jun, 2009.

NIELSEN, J. Projetando Websites. Rio de Janeiro: Elsevier, 2000.

PREECE, Jennifer; ROGERS, Yvonne; SHARP, Helen. Design de interação: além da interação homem-computador. Porto Alegre: Bookman, 2005.

ROCHA, Heloisa Vieira da, BARANAUSKAS, Maria Cecília C. Design e avaliação de interfaces humano-computador. São Paulo: NIED/UNICAMP, 2003.

RUBIN, Jeffrey; CHISNELL, Dana. Handbook of usability: how to plan, design, and conduct effective tests. 2ed. Indianapolis: Wiley Publishing, 2008.

TULLIS, Thomas, ALBERT, William. Measuring the user experience: collecting, analyzing and presenting usability. USA: Morgan Kaufmann, 2008. 